\title{
Proceso de la Planeación Estratégica
}

Compilación comentada y subrayada por el autor

Lic. Fernando MÁLAGA MÁLAGA

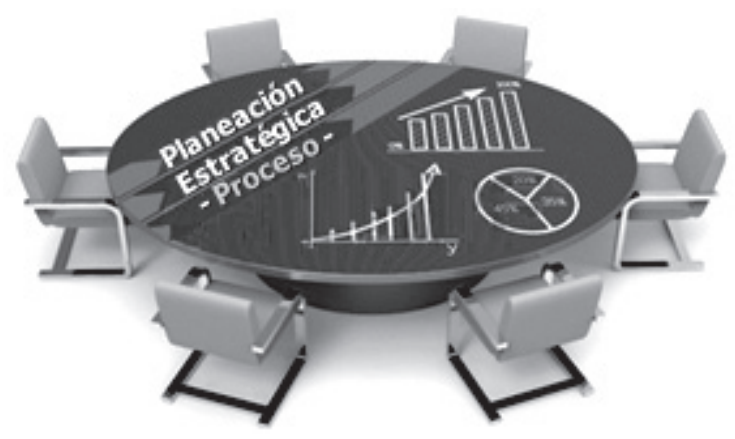

\section{RESUMEN}

Una estrategia corporativa, además de elegir el plan adecuado para el crecimiento y desarrollo organizacional de las empresas en el mundo de hoy, debe también asegurar que la empresa puede reaccionar rápida y adecuadamente a los mercados cada vez más dinámicos y en sus entornos de alta competitividad.

Las perspectivas y características de esta planeación, así como las estrategias para el cambio frente a las dificultades o cierto rechazo que toda nueva estrategia pudiera encontrar para implementar estos cambios serán examinadas en este ensayo, de acuerdo a las doctrinas de los más clásicos tratadistas al respecto, extraídas en compilación comentada.

PALABRAS CLAVE: estrategia, procesos, el cambio, factores y modos, planeamiento, estrategia corporativa.

\section{SUMMARY}

Corporate strategy, in addition to choosing the right plan for organizational growth and development of businesses in today's world, must also ensure that the company can react quickly and appropriately to the increasingly dynamic markets and its highly competitive environments.

The perspectives and characteristics of this planning, as well as strategies for changing the face of difficulties or a rejection that any new strategy could find 
to implement these changes will be examined in this paper, according to the doctrines of the classic writers about, taken in annotated compilation.

KEY WORDS: strategy, processes, the change, factors and methods, planning, corporate strategy.

\section{TEMARIO}

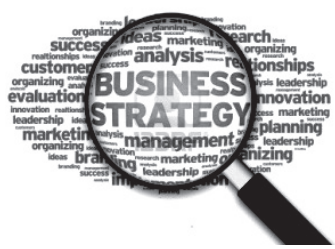

\section{Perspectivas y Características}

2. Estrategias Genéricas

3. Factores y Modos Estratégicos

4. Estrategia Corporativa y de Negocios

5. Estrategia para el Cambio

\section{PERSPECTIVAS Y CARACTERÍSTICAS}

El proceso de la estrategia tiene diversas perspectivas. En términos abiertos, podría ser considerado como un programa general diseñado para definir y alcanzar los objetivos de la organización y poner en práctica su misión, implicando para ello la capacidad de tener un papel modernamente activo, racional y bien definido, conceptualmente. Este plano distribuye el peso conceptual, entre la misión y pensamiento estratégicos.

Otra perspectiva en el proceso estratégico es poder contar con un patrón de las respuestas de la organización a su ambiente, a través del tiempo. Es decir, toda organización tiene una relación con su medio ambiente, la que puede ser examinada y descrita.

Las perspectivas estratégicas pueden resumirse en las cinco características fundamentales que reseñan Robert H. Hayes y Steve C.Wheelwright(1991:420):

1) “Horizonte temporal. Se emplea para describir actividades que comprenden un amplio horizonte de tiempo, en relación con el tiempo que se tarda en efectuarlas y el que se tarda en observar su impacto.

2) Impacto. Pese a que las consecuencias de seguir una estrategia determinada tal vez no se hagan evidentes durante largo tiempo, su impacto final será importante. 
3) Concentración de esfuerzo. Una estrategia eficaz por lo general suele requerir concentrar la actividad, esfuerzo o atención en un número bastante reducido de fines.

4) Patrón de decisiones. Aunque algunas compañias necesitan hacer sólo algunas decisiones importantes a fin de poner en práctica su estrategia seleccionada, la mayor parte de las estrategias exigen que ciertos tipos de decisión sean tomados con el tiempo.

5) Capacidad de penetración. Una estrategia engloba un amplio espectro de actividades, las cuales incluyen desde los procesos de asignación de recursos hasta las operaciones diarias. Además, la necesidad de congruencia a través del tiempo, en estas actividades, requiere que todos los niveles de una organización actúen, casi indistintamente, en formas que refuercen la estrategia".

Estas cinco características indican, claramente, que la estrategia de una organización constituye el eje central en torno al cual giran otras actividades principales de ella. La estrategia es a largo plazo y de gran alcance; engloba y controla las acciones más importantes de la organización siendo un determinante básico de su éxito o fracaso.

\section{ESTRATEGIAS GENÉRICAS}

Las estratégicas genéricas están basadas en el principio que indica que el diseño general de una organización puede ser descrito a través del logro de los objetivos, políticas, y métodos, para llegar a un diseño integral, el cual debe proporcionar a la

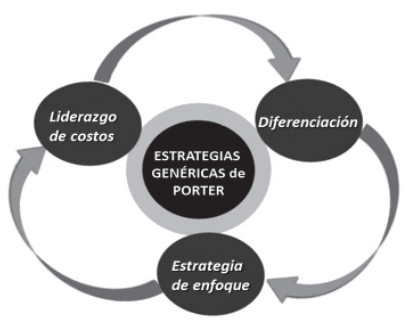
empresa, ventajas sobre su mercado. Michael Porter, hacia 1980 sentó las bases para estos conceptos, partiendo de los tres enfoques competitivos siguientes:

1. “Liderazgo de costos. Es tal vez la más clara de las estrategias genéricas: ...ser el productor de menor costo en su sector. Las fuentes de las ventajas en el costo... pueden incluir la persecución de las economías de escala, tecnología propia, acceso preferencial a materias primas y otros factores.

2. Diferenciación. En una estrategia de diferenciación, la empresa busca ser única en su sector industrial junto con algunas dimensiones que son ampliamente valoradas por los compradores.Puede basarse en el producto mismo, el sistema de entrega por medio del cual se vende, el enfoque de mercadotecnia y un amplio rango de factores más. 
3. Estrategia de enfoque. Descansa en la elección de un panorama de competencia muy estrecho... El enfocador busca lograr una ventaja competitiva en sus segmentos blanco (target), aunque no posea una ventaja competitiva general"'.

Según Porter, las estrategias de liderazgo de costo y de diferenciación tienen como objetivo amplio todo el mercado, mientras que la estrategia genérica de enfoque limita su objetivo solamente a un segmento o nicho.

Finalmente, Porter amplía el concepto de esta última estrategia, mencionando que consta de dos variantes: "en el 'enfoque de costo' una empresa busca una ventaja de costo en su segmento blanco... (explota las diferencias en el comportamiento de costos), mientras que en el 'enfoque de diferenciación' una empresa busca la diferenciación en su segmento blanco... (explota las necesidades especiales de los compradores en ciertos segmentos) "'.2.

¿Cómo una compañía, basada en la propuesta de Porter, puede obtener ventajas competitivas? Robbins y De Cenzo nos ofrecen alternativas para diseñar los proyectos clave y los planes de acción futuros, en estos "detalles sobre un modelo administrativo":

\section{A. "Ventaja en los costos:}

- Eficiencia de operaciones

- Economía de escala

- Innovación tecnológica

- Mano de obra a bajo costo

- Acceso preferencial a la materia prima

\section{B. Estrategia de Diferenciación:}

- Gran calidad

- Servicio excelente

- Diseño innovador

- Capacidad tecnológica

- Imagen extraordinariamente positiva de la marca

C. Estrategia del Punto Central: (Enfoque)

- Variedad del producto

- Tipo de comprador final

- Canal de distribución

- Ubicación geográfica de los compradores"3.

1 Porter, Michael E.: "Ventaja Competitiva" - CECSA, 1995:30/32.

2 Ibid. Porter, págs. 32/33.

3 Robbins Stephen y De Cenzo David A.: “Fundamentos de Administración” - 1991:74. 
"Tomando como base los análisis de Porter, varios autores desarrollan el tema de la estrategia competitiva. Un enfoque interesante es cómo analizar a un competidor, cuál será su perfil de respuesta y cuál su tiempo de respuesta, comentan Hermida y Serra, quienes nos aconsejan que, el no elegir ninguno de los tres enfoques alternativos y tomar una decisión estratégica diferente, es lo que Porter llama estancado en el medio, que conduce al negocio a una pobre situación estratégica diferente"4.

\section{FACTORES Y MODOS ESTRATÉGICOS}

Los factores estratégicos son los procesos políticos de la alta dirección y las reacciones individuales internas de la organización que pueden forzar la revisión de la estrategia. Los modos de formular una estrategia general han sido resumidos en tres, descritos por Mintzberg, como el modo emprendedor o empresarial, el adaptativo y el de planeación ${ }^{5}$.

En el modo emprendedor la característica es un líder fuerte, generalmente fundador de la empresa, que toma decisiones atrevidas y riesgosas más o menos intuitivamente; es decir, se basa en su juicio personal que es fruto de la experiencia. Desde esta perspectiva, la visión empresarial "está basada en conocimientos íntimos y detallados del negocio... la estrategia no es un plan en papel, sino una visión en la mente”.

Al modo adaptativo se le conoce con el nombre de "Ciencia de la Improvisación". A diferencia del emprendedor, quien afronta el ambiente con una fuerza que necesita ser controlada, a este modo se le critica por ser demasiado defensivo ante las acciones de los competidores. Típicamente, "la empresa reacciona ante los problemas existentes, en lugar de buscar nuevas oportunidades"

El modo de planeación es el tercer modo de formulación de estrategia general según Mintzberg. Bajo esta óptica, los planificadores siguen un procedimiento sistemático que los obliga a analizar el ambiente, "la evaluación de los costos y los beneficios de las propuestas que compiten" buscando la integración de la organización, para elaborar un plan futuro.

Los planificadores deben también tomar decisiones arriesgadas, pero siempre sus decisiones son sistemáticas y estructuradas, basadas en una estimación racional de las oportunidades y de las amenazas del ambiente, debiendo siempre ser congruentes con la misión y las capacidades globales de la organización.

4 Hermida J. y Sierra R.: “Administración Estratégica. Un Enfoque Competitivo y Emprendedor”-Macci, 1989:288/289.

5 Mintzberg, Henry; Quinn, James Brian y Voyer, John: “El ProcesoEstratégico”1997:26 (Condensado por autor). 
4. ESTRATEGIA CORPORATIVA Y DE NEGOCIOS

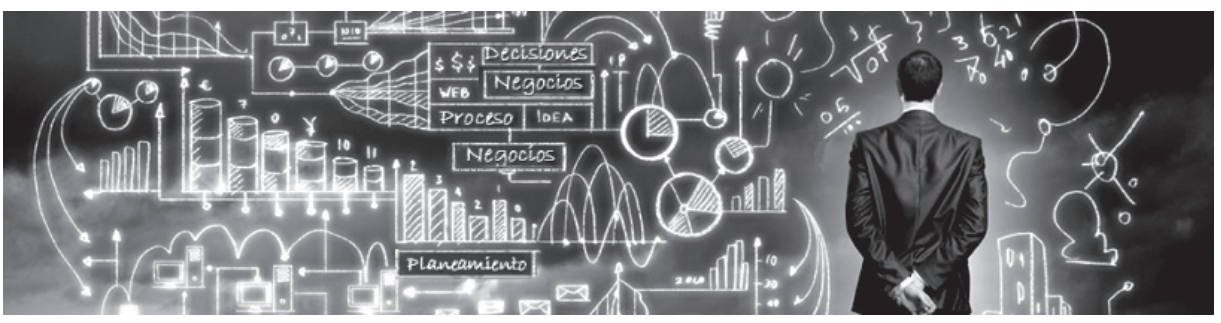

"La eficiencia es hacer las cosas bien: la efectividad es hacer cosas debidas. Esta frase atribuida a Peter Drucker, hace hincapié en la importancia de enfocarse en las áreas debidas, en las que se invertirán tiempo, energía, talento y otros recursos a fin de lograr resultados. Muchas empresas trabajan con demasiado afán para ser eficientes en cosas que probablemente ni siquiera haría falta que se hicieran" $"$.

La estrategia del primer nivel corporativo, se centra en la formulación de la alta administración con el fin de supervisar los intereses y las operaciones diversas en esas precisas "áreas debidas" de todas aquellas corporaciones que manejan diversas líneas. Peter Drucker plantea, sobre la base de proyecciones hipotéticas, un método en el que "una teoría del negocio tiene tres partes:

- La primera, se hacen supuestos acerca del ambiente de la organización: la sociedad y su estructura, el mercado, el cliente y la tecnología.

- La segunda, se hacen supuestos sobre la misión específica de la organización. No es necesario que la misión de una organización sea tan ambiciosa.

- La tercera, se hacen supuestos acerca de las competencias centrales que se necesitan para que la organización realice su misión.

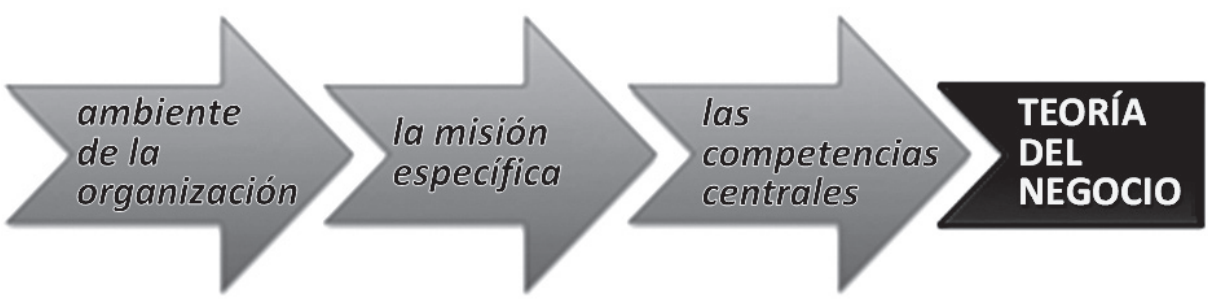

Desde luego, todo esto suena engañosamente sencillo. Habitualmente se necesitan años de arduo trabajo, de pensar y de experimentar para llegar a una

6 Morrisey,George L.: “Planeación Táctica” - Prentice-Hall 1996:27. 
teoría del negocio que sea clara, consecuente y válida. Para tener éxito, toda organización tiene que formular una teoría" "7.

Por ello, las principales preguntas que se debe responder a este nivel son: ¿en qué tipos de negocios se debe involucrar la compañía?, ¿cuáles son las metas y las expectativas para cada negocio?, ¿cómo se deben asignar los recursos para que se puedan alcanzar las metas previstas en el proceso de planificación?

\section{ESTRATEGIA PARA EL CAMBIO}

Las estrategias están enfocadas de acuerdo a un plan y a su aplicación, de tal modo que sólo se tropiece con un mínimo de dificultades por las reacciones de las personas a quienes afecten los cambios. De acuerdo con el rol de planeación empresarial, se debe apuntalar la estrategia paralelamente con los cursos de acción que se implantan después de haber tomado en consideración las contingencias previsibles y sobre todo, la conducta de los demás involucrados.

Las amplias tendencias económicas que crean trastornos globales por encima de las fronteras, serán influyentes en los años venideros para el éxito o fracaso de los negocios. Aquí es oportuno sintetizar las precisiones que sobre las dimensiones para el manejo del cambio estratégico, nos predice Ohmae:

1) un cambio de las industrias con alto grado de mano de obra a otras industrias con alto grado de capital;

2) un cambio de las compañias multinacionales a multilocales;

3) un cambio en el índice entre costos fijos y costos variables en varias industrias;

4) un cambio en las industrias con base en el acero a las industrias con base en la electrónica (caso japonés);

5) un cambio en la definición de la unidad de negocios;

6) un cambio en la administración financiera de internacional a local;

7) un cambio hacia un sistema coordinado de valores corporativos.

Un conocimiento de estos acontecimientos puede agregar una vital dimensión de realismo al pensamiento del estratega y proporcionar una perspectiva global a su tarea de planeación" .

7 Drucker, Peter F.: “Su visión sobre: La Administración. La Organización basada en la Información. La Economía. La Sociedad” - Norma 1996:29/30.

8 Ohmae, Kenichi: "La Mente del Estratega" - McGraw-Hill, 1989:200. 
"Los últimos años han presenciado la decadencia, una por una de empresas que en un tiempo eran dominantes: General Motors e IBM, por nombrar sólo unas pocas, critica Drucker, señalando que en todos los casos, la causa principal fue por lo menos uno de los que califica como los cinco pecados mortales de los negocios. Veamos cuáles son éstos, para tomar las providencias estratégicas del caso.

1. El primero de los pecados mortales y, seguramente el más común, es la adoración de los altos márgenes de utilidades y de los precios de prima.

2. Se relaciona intimamente con este primer pecado el segundo: fijar mal el precio de un nuevo producto cobrando 'lo que resista el mercado'.

3. El tercer pecado mortal es fijar precios basándose en los costos. Lo único que funciona es fijar costos basándose en los precios.

4. El cuarto de los pecados mortales de los negocios es sacrificar las oportunidades de mañana en el altar de ayer. Esto fue lo que hizo descarrilar a IBM.

5. El último de los pecados mortales es alimentar los problemas y dejar morir las oportunidades".

Gary Hamel, coautor con C.K. Prahalad del libro guía sobre estrategia corporativa: "Competing for the Future", escribió un decálogo sobre innovación y estrategia en la prestigiosa revista de Harvard concluyendo, acertadamente, que la formulación estratégica es un proceso de descubrimiento y de inventiva. ${ }^{10}$

Internalizar la sabiduría y experiencias de Kenichi Ohmae y Peter Drucker, sería el primer cambio estratégico en nuestros propios enfoques y pensamientos. Una lectura más amplia, debe llevarnos a profundos análisis para así diseñar un proceso estratégico más maduro y lograr administrar la planeación más sólidamente.

9 Ibid. Drucker, págs. 45/47/48/49.

10 http://garyhamelotroguru.blogspot.com/2009/05/gary-hamel-mi-guru-estrategia-e. $\underline{\mathrm{html}}$. 